DR. ALBERTO AIMO (Orcid ID : 0000-0001-9129-9519)

Article type : Original Paper

\title{
A simple echocardiographic score to rule out cardiac amyloidosis
}

Alberto Aimo, $\mathrm{MD}^{1^{*}}$, Vladyslav Chubuchny, $\mathrm{MD}^{2 *}$, Giuseppe Vergaro, $\mathrm{MD}, \mathrm{PhD}^{1,2}$, Andrea Barison, $\mathrm{MD}, \mathrm{PhD}^{1,2}$, Martin Nicol, $\mathrm{MD}^{3}$, Alain Cohen Solal, $\mathrm{MD}, \mathrm{PhD}^{3}$, Vincenzo Castiglione, $\mathrm{MD}^{1}$, Valentina Spini, $\mathrm{MD}^{2}$, Alberto Giannoni, $\mathrm{MD}, \mathrm{PhD}^{1,2}$, Christina Petersen, $\mathrm{MD}^{2}$, Claudia Taddei, BSc${ }^{2}$, Emilio Pasanisi, $\mathrm{MD}^{2}$, Liza Chacko, $\mathrm{MD}^{4}$, Raffaele Martone, $\mathrm{MD}^{4}$, Dan Knight, $\mathrm{MD}^{4}$, James Brown, MA, MB${ }^{4}$, Ana Martinez-Naharro, $\mathrm{MD}^{4}$, Claudio Passino, $\mathrm{MD}^{1,2}$, Marianna Fontana, $\mathrm{MD}, \mathrm{PhD}^{4}$, Michele Emdin, $\mathrm{MD}, \mathrm{PhD}^{1,2}$

1. Institute of Life Sciences, Scuola Superiore Sant'Anna, Pisa, Italy; 2. Cardiology Division, Fondazione Toscana Gabriele Monasterio, Pisa, Italy; 3. Cardiology Department, Hopital Lariboisiere, 2 rue Ambroise Paré, Paris, France; 4. National Amyloidosis Centre, University College London, Royal Free Campus, London, UK. *These Authors equally contributed.

Short title: An echocardiographic score for cardiac amyloidosis.

Word count: 2910 (text)

Conflicts of interest: none.

Address for correspondence:

Alberto Aimo, MD

Institute of Life Sciences, Scuola Superiore Sant'Anna

Piazza Martiri della Libertà 33

Phone +393477084391

This article has been accepted for publication and undergone full peer review but has not been through the copyediting, typesetting, pagination and proofreading process, which may lead to differences between this version and the Version of Record. Please cite this article as doi: $\underline{10.1111 / \text { ECI.13449 }}$

This article is protected by copyright. All rights reserved 


\begin{abstract}
Background: Early diagnosis of cardiac amyloidosis (CA) is warranted to initiate specific treatment and improve outcome. The amyloid light chain (AL) and inferior wall thickness (IWT) scores have been proposed to assess patients referred by hematologists or with unexplained left ventricular (LV) hypertrophy, respectively. These scores are composed of 4 or 5 variables, respectively, including strain data.
\end{abstract}

Methods: Based on 2 variables common to the AL and IWT scores, we defined a simple score named AMYLoidosis Index (AMYLI) as the product of relative wall thickness (RWT) and E/e' ratio, and assessed its diagnostic performance.

Results: In the original cohort $(\mathrm{n}=251)$, CA was ultimately diagnosed in 111 patients $(44 \%)$. The 2.22 value was selected as rule-out cut-off (negative likelihood ratio [LR-] 0.0). In the hematology subset, AL CA was diagnosed in 32 patients (48\%), with 2.36 as rule-out cut-off (LR- 0.0$)$. In the hypertrophy subset, ATTR CA was diagnosed in 79 patients (43\%), with 2.22 as the best rule-out cut-off (LR- 0.0). In the validation cohort $(n=691)$, the same cut-offs proved effective: indeed, there were no patients with CA in the whole population or in the hematology or hypertrophy subsets scoring $<2.22,<2.36$ or $<2.22$, respectively.

Conclusions: The AMYLI score (RWT* E/e') may have a role as an initial screening tool for CA. $\mathrm{A}<2.22$ value excludes the diagnosis in patients undergoing a diagnostic screening for $\mathrm{CA}$, while a $<2.36$ and a $<2.22$ value may be better considered in the subsets with suspected cardiac AL amyloidosis or unexplained hypertrophy, respectively.

Word count: 249 (abstract)

Keywords: cardiac amyloidosis, diagnosis, echocardiography, score, RWT, E/e'. 


\section{Introduction}

Amyloidosis is a systemic disorder characterized by the extracellular deposition of circulating proteins into amyloid fibers. In the 2 most common forms of amyloidosis, these proteins are monoclonal light chains (AL amyloidosis), or transthyretin (ATTR amyloidosis) [1].

Cardiac involvement is common in both AL and ATTR amyloidosis, predicts a worse outcome, and has important implications for treatment [1]. The diagnostic work-up starts with the identification of clinical features, imaging and electrocardiographic findings suggestive or compatible with cardiac amyloidosis (CA). Following the introduction of a novel algorithm by Gillmore et al., ATTR CA can be diagnosed non-invasively when there is a moderate to intense diphosphonate uptake at a scintigraphy scan (Perugini score 2-3), and there is no monoclonal gammopathy. In all other cases, the histological demonstration of amyloid accumulation is required [2]. For example, a definite diagnosis of AL CA still requires the detection of amyloid deposits within the heart, the abdominal fat pad, or another organ, in the setting of a monoclonal gammopathy [3].

Transthoracic echocardiogram is a commonly available, first-line examination in patients with a suspicion of cardiac disease [4]. The most common findings are increased left ventricular (LV) wall thickness with normal or small LV cavity size, and left atrial or biatrial dilatation secondary to diastolic dysfunction, and sometimes right ventricular dysfunction; a more specific finding is the better contractility of apical segments compared to basal segments [4]. Very recently, Boldrini et al. have proposed 2 echocardiographic scores named AL score and increased wall thickness (IWT) score, to be used in patients evaluated for blood dyscrasia and with a clinical suspicion of CA, or in patients with unexplained LV hypertrophy, respectively [5]. These scores include relative wall thickness (RWT), E/e' ratio, tricuspid annular plane systolic excursion (TAPSE), global longitudinal strain (GLS), and, in the IWT score, also the systolic septal longitudinal apexto-base (SAB) strain gradient (Supplemental Table 1). These scores were defined in a pooled cohort of patients from 3 international referral centers for $\mathrm{CA}$, based on independent predictors of diagnosis and their relative weights. The AL and IWT scores displayed good diagnostic accuracy, and could then help clinicians decide whether or not to perform further diagnostic tests, such as cardiac magnetic resonance (CMR) or diphosphonate scintigraphy, and up to endomyocardial biopsy [5]. Possible drawbacks of these scores are the need for strain assessment, which is not currently part of the usual echocardiographic approach, the different equations to calculate the 2 scores, and the absence of clearly defined cut-offs to rule-out and rule-in CA. 
In the present study we tried to overcome these limitations by creating a simpler score to diagnose either AL or ATTR amyloidosis, including just 2 commonly available variables (RWT and E/e' ratio), and by identifying setting-specific cut-offs to rule out CA.

\section{Materials and methods}

\section{Creation of the AMYLI score}

The AL and IWT scores were defined in a cohort of 1187 patients from 3 referral centers in London, Pisa, and Madrid [5]. Boldrini et al. introduced 2 diagnostic scores to be used in the corresponding settings: the AL score (RWT: $2 *$ IWT in end-diastole/LV end-diastolic diameter]; E/e' ratio; TAPSE; GLS), and the IWT score (all the variables above plus SAB ratio) (Supplemental Table 1) [5]. We selected 2 commonly available variables reflecting a disproportionate increased wall thickness relatively to LV volume (RWT) and diastolic dysfunction (E/e' ratio), which are common features of cardiac involvement in CA. The AMYLoidosis Index (AMYLI) score was then defined as the product of RWT and E/e'.

\section{Original cohort}

Consecutive patients referred for suspected CA between 2009 and 2019 at a tertiary referral center in Italy (Fondazione Toscana Gabriele Monasterio, Pisa, Italy) were evaluated. Among the 340 patients, those with conditions making the E/e' ratio unreliable (atrial fibrillation or flutter, tachycardia with fusion of $\mathrm{E}$ and $\mathrm{A}$ velocities, moderate to severe mitral regurgitation or aortic regurgitation, mitral valve repair or replacement, severe mitral annular calcification, significant mitral stenosis, or left bundle branch block) [6] or lack of RWT and E/e' ratio were excluded. The remaining patients $(n=251,74 \%)$ were categorized in 2 groups: those referred by specialized hematologists because of suspected cardiac involvement in AL amyloidosis ( $n=67,27 \%$ ), and those with increased LV wall thickness on echocardiogram (interventricular septal thickness or IWT in end-diastole $\geq 12 \mathrm{~mm}$ ) that had suggested the possibility of CA ( $\mathrm{n}=184,73 \%$ ) [5]. From here on, these 2 subsets will be referred to as "hematology subset" and "hypertrophy subset", respectively.

Patients underwent a complete diagnostic work-up including clinical evaluation, echocardiography, CMR when clinically indicated, serum and urine biochemistry comprising Nterminal pro-B-type natriuretic peptide, serum free light chain assay along with serum and urine immunofixation-electrophoresis and myocardial or non-myocardial biopsy [5]. Patients with 
suspected ATTR cardiomyopathy also underwent diphosphonate scintigraphy. The study protocol conformed to the 1975 Declaration of Helsinki [7], and was approved by the Institution's human research committee. All patients provided written informed consent.

\section{Diagnosis of cardiac amyloidosis}

CA was diagnosed according to validated protocols [2,8]. Patients with HF symptoms or other symptoms possibly attributed to cardiac disease (such as syncope or bradyarrhythmia) plus clinical, electrocardiographic, biohumoral or imaging (echocardiography and/or cardiac magnetic resonance - CMR) findings deemed compatible or suggestive of CA by expert readers underwent a validated diagnostic protocol including diphosphonate scintigraphy, the search for a monoclonal protein in the serum and urine, and biopsy of cardiac or extracardiac tissues when needed to confirm the diagnosis. Cardiac AL amyloidosis was diagnosed in the 2 following cases: 1) the combination of typical features on CMR and histologically proven systemic AL amyloidosis using a non-cardiac biopsy $(\mathrm{n}=18,56 \%)$ [8], or 2$)$ an endomyocardial biopsy containing AL amyloid $(\mathrm{n}=15,45 \%)$. Cardiac ATTR amyloidosis was diagnosed with 1) grade 2 or 3 cardiac uptake on diphosphonate scintigraphy in the absence of monoclonal gammopathy $(n=37,56 \%)$, or 2$)$ a cardiac biopsy containing ATTR amyloid $(n=29,44 \%)$ [2]. The details of echocardiographic evaluation are reported in the Supplemental Material.

\section{Validation cohort}

For score validation, 1044 consecutive patients undergoing a diagnostic workup for CA at the National Amyloidosis Centre, London, United Kingdom or at the Hôpital Lariboisière, Paris, France were considered. After excluding 353 patients meeting exclusion criteria (34\%), the validation cohort was composed of 691 patients evaluated for suspected CA at the National Amyloidosis Centre, London, United Kingdom ( $\mathrm{n}=611,88 \%$ ), or at the Hôpital Lariboisière, Paris, France $(\mathrm{n}=80,12 \%) . \mathrm{x}$

\section{Statistical analysis}

The statistical analysis was performed using IBM SPSS Statistics (version 22, 2013) and R statistical software (http://www.r-project.org/, version 3.4.4). Normal distribution was assessed through the Kolmogorov-Smirnov test; variables with normal distribution were presented as mean \pm standard deviation, while those with non-normal distribution as median and interquartile 
interval. Mean differences among groups were evaluated through the unpaired Student $\mathrm{T}$ test or the Mann Whitney U test, as appropriate. Categorical variables were compared by the Chi-square test with Yates correction. The performance of diagnostic scores was evaluated in terms of area under the curve (AUC) at receiver operating characteristics (ROC) analysis, and ROC curves were compared through the DeLong's test. As a decisional criterion for optimal rule-out cut-off, a negative likelihood ratio (LR-: [1-sensitivity]/specificity) $<0.1$ was considered [7]; the AMYLI score value with the highest LR- below the 0.1 threshold was selected. The value with the highest LR+ value (sensitivity/[1-specificity]) was selected as a rule-in cut-off, as no value met the criterion for an optimal rule-in cut-off $(\mathrm{LR}+>10)$ [9]. Sensitivity, specificity, positive and negative predictive values (PPV/NPV) of each proposed cut-off were calculated. $\mathrm{p}$ values $<0.05$ were considered statistically significant.

\section{Results}

\section{Original cohort}

Patient characteristics of the original cohort are summarized in Table 1. Patients were aged 79 years (74-84, 63\% males). A mild systolic dysfunction was observed, with median LV ejection fraction (LVEF) 47\%, and GLS -13\%. The RWT was 0.52 (0.44-0.65); diastolic dysfunction (median E/e' 12.6), and preserved right ventricular function (median TAPSE $18 \mathrm{~mm}$ ) were also observed. Patients evaluated for unexplained hypertrophy were older, had higher arterial pressure values and LV mass index, and lower LVEF (Table 1).

AL CA was diagnosed in 32 patients (48\% of the hematology subset), and ATTR CA in 79 patients (43\% of the hypertrophy subset). The disorders diagnosed in patients with no CA are reported in Supplemental Table 2.

\section{Validation cohort}

Baseline characteristics of the validation cohort are reported in Table 2. Compared to the original cohort, these patients were younger, and more often belonged to the hematology subset. Another difference was represented by higher AMYLI score values in the hematology subset. Furthermore, more patients were found to have CA: in the hematology subset, cardiac AL amyloidosis was diagnosed in 385 (94\%) patients; in the hypertrophy subset, cardiac ATTR amyloidosis was 
diagnosed in 193 (68\%) patients. . The differential diagnoses are reported in Supplemental Table 2.

\section{Whole original and validation cohorts: AMYLI score values}

In the entire original cohort, patients with CA had a significantly higher AMYLI score value (9.8 [6.6-13.7] vs. 4.9 [3.6-7.4]; $\mathrm{p}<0.001$ ) (Figure 1), with a 0.790 AUC. AMYLI score values did not differ significantly between the hematology and hypertrophy subsets (Table 1).

In the entire validation cohort, the AMYLI score had an AUC of 0.572. In the hematology subset $(n=408)$, all variables needed to calculate the AL score were available in $67 \%$ of patients only, and the IWT score could be calculated in $81 \%$ of the 283 patients from the hypertrophy subset. In the hematology subset, AUC values for the AL score and the AMYLI score were quite low ( 0.625 vs. 0.513$)$, but without significant differences $(p=0.736)$. In the hypertrophy subset, the IWT score had a 0.632 AUC value and the AMYLI score a 0.655 AUC $(\mathrm{p}=0.853)$.

\section{Whole original and validation cohorts: rule-out cut-off}

As a rule-out cut-off for the diagnosis of CA, the AMYLI score value of 2.22 as having a LR- of 0.0 in the original cohort. In the validation cohort it had a $\mathrm{LR}=\infty$ as no patients scoring $<2.2 \mathrm{had}$ CA (Table 3).

\section{The AMYLI and AL scores to diagnose cardiac AL amyloidosis}

In the hematology subset of the original cohort $(n=67)$, the AMYLI score had a 0.847 AUC. The score value with the lowest LR- (2.36, LR- 0.00) was selected (Table 3). In the validation cohort, no patient with an AMYLI score $<2.36$ had cardiac AL amyloidosis (Figure 2).

In the original cohort, the AMYLI score was calculated in all patients, while all variables needed to calculate the AL score were available in just 41 patients (61\%). The diagnostic performance of the 2 scores, as evaluated through the ROC curves, did not differ significantly (Figure 3).

\section{The AMYLI and IWT scores to diagnose cardiac amyloidosis}

In the hypertrophy subset of the original cohort, the IWT score could be calculated just in $57 \%$ of patients, and the diagnostic performances of the AMYLI and IWT scores were not significantly 
different (Figure 3). The 2.22 score value was selected as rule-out cut-off, with a good performance in the validation cohort (Table 3).

\section{Discussion}

In the present study we introduce a score for the initial screening of CA, which includes 2 commonly available echocardiographic parameters, i.e. RWT and E/e' ratio. We report that the diagnostic performance of the AMYLI score is non-inferior to the more complex AL and IWT scores, and that rule-out cut-offs are particularly effective to exclude a diagnosis of CA, thus avoiding the need for further investigations, possibly including endomyocardial biopsy. Specifically, we considered 2 cohorts: an original cohort including 251 patients, and a validation cohort composed of 691 patients examined in other 2 specialized centers. A very different prevalence of CA was observed in the 2 cohorts ( $44 \%$ vs. $84 \%$, respectively), likely reflecting different policies of patient referral, as the diagnostic algorithm was standardized. The cohorts were also heterogeneous with respect to the reason for referral, namely a blood dyscrasia with possible cardiac AL amyloidosis ("hematology subset": $27 \%$ in the original cohort vs. $59 \%$ in the validation cohort), or unexplained LV hypertrophy ("hypertrophy subset": $73 \%$ in the original cohort vs. $41 \%$ in the validation cohort). In agreement with the different observed prevalence of CA in the 2 cohorts, the AMYLI score had higher AUC values in the original than the validation cohorts, both in the whole population and in the hematology and hypertrophy subsets. Nonetheless, AUC values in the 2 subsets were not significantly different than the AL or IWT scores, respectively, denoting that the diagnostic performance of both the AMYLI and the AL or IWT scores were similarly affected. As the next step, we calculated rule-out cut-offs, corresponding to the AMYLI score values associated with LR- $<0.1$ in both cohorts. These cutoffs were 2.22 in the 2 whole cohorts, 2.36 in the hematology subsets, and 2.22 in the hypertrophy subsets. CA could then be reliably excluded when AMYLI score values are lower these values in the corresponding settings. The conclusion that the AMYLI score is a useful tool to excludeCA seems reasonable if we consider that cardiac involvement is likely absent when both RWT (denoting -pseudo-hypertrophy disproportionate to LV volumes) and E/e' (reflecting diastolic dysfunction) are low.

Our score was developed based on the AL and IWT scores, which included independent predictors of CA among a range of echocardiographic variables. We choose to include only RWT 
and E/e' ratio, and not TAPSE (as right ventricular involvement typically develops in a more advanced stage), or measures requiring strain analysis, usually not performed in clinical practice, because of several constraints (lack of a dedicated software, need for an operator learning curve, additional time needed, or poor image quality). In agreement with the better feasibility of the AMYLI score, the AL and IWT score could be retrospectively calculated only in $61 \%$ and $57 \%$ of patients in the original cohort, respectively, and in $75 \%$ and $70 \%$ of patients in the validation cohort, respectively,. In particular, despite being independently associated to patients' prognosis in AL amyloidosis [10], RWT represents a quite simplistic parameter of LV geometry, and far more complicated parameters might be needed to track all the different hypertrophic patterns of CA $[11,12]$. Contrary to the AL and IWT scores, we also not assigned a coefficient to the 2 variables selected, and we tested the same score (RWT*E/e') in the whole population as well as the hematology and hypertrophy cohort. Furthermore, while Boldrini et al. had calculated the diagnostic performance of each score point [5], we tried to clearly define rule-out cut-off values to be proposed for use in current clinical practice. As discussed above, the rule-out cut-offs proved effective for excluding CA. We may conclude that when the product of RWT and E/e' is $<2.36$ in a patient referred by the hematologist for suspected cardiac AL amyloidosis, $<2.22$ in a patient with hypertrophy with no obvious causes, or again $<2.22$ in a patient undergoing a diagnostic workup for CA, the diagnosis can be safely excluded, and further investigations (diphosphonate scintigraphy, search for a monoclonal protein and cardiac magnetic resonance if not already performed, and histological examinations) are then not required. A possible exception is represented by patients with elevated NT-proBNP or high-sensitivity troponins; in these cases, the cause of the ongoing myocardial damage (either CA or other disease processes) should be searched [13].

Several other limitations can be identified. First, the variables to be included in the AMYLI score were arbitrarily selected from the 4 or 5 variables in the AL and IWT scores, respectively, and the choice to multiply these variables instead of, for example, giving them a differential weight and summing them was again arbitrary. Second, the study population was retrospectively analyzed, and partially overlapping with the one used to create the AL and IWT scores; nevertheless, the analysis of the AMYLI score was performed independently from and blinded to the previous scores. Third, as stated above, the prevalence of CA was quite high, particularly in the validation cohort, reasonably because of the referral of patients with a higher pre-test probability of CA in London and Paris compared to Pisa. Further larger studies are needed to 
validate the AMYLI score in a wider, more general population, with a lower pre-test probability of CA. On the other hand, sensitivity and specificity, which are used to calculate LR- values and were then used to define rule-out cut-offs, are not affected by disease prevalence [7]. The proposed cut-offs can then be reliably evaluated regardless of the strength of the suspicion of CA. Moreover, even though cardiologists were not blinded to the suspicion of amyloidosis, because they worked in amyloidosis specialized hospitals, they were blinded to the score (which had not been defined a priori) and to the final diagnosis of amyloidosis (which relied on other diagnostic modalities, according to Gilmore's algorithm) [2]. Fourth, to meet the criteria for optimal rule-out cut-off (LR- $<0.1)$, very low AMYLI score were selected, which means that CA could be ruled out only in a small proportion of patients (5\% in the original cohort, $2 \%$ in the validation cohort). Fifth, our conclusion that further diagnostic investigations should be performed in patients with AMYLI score values lower than rule-out cut-offs, but elevated biomarkers (NT-proBNP or highsensitivity troponins) is not based on our results, and should be evaluated in prospective studies with larger population sizes (to account for the low number of patients with AMYLI score below rule-out cut-offs).

\section{Conclusions}

We propose the AMYLI score (RWT* E/e') as a possible initial screening tool for CA. An AMYLI score value $<2.22$ excludes the diagnosis in patients undergoing a diagnostic screening for $\mathrm{CA}$, while values $<2.36$ and $<2.22$ may be better considered in the subsets with suspected cardiac AL amyloidosis or unexplained hypertrophy, respectively.

\section{Figure legends}

Figure 1. Original cohort: AMYLoidosis Index (AMYLI) score values among patients with or without cardiac amyloidosis. 
Patients diagnosed with cardiac amyloidosis were 111 out of 251 (44\%). RWT, relative wall thickness.

Figure 2. AMYLoidosis Index (AMYLI) score values among patients with or without amyloid light chain (AL) or amyloid transthyretin (ATTR) cardiac amyloidosis.

Patients referred by hematologists ("hematology subset") and those evaluated for unexplained hypertrophy ("hypertrophy subset") were analyzed in both the original and validation cohorts. The rule-in and rule-out cut-offs are reported in the graphs.

Figure 3. Area under the curve (AUC) values of the AMYLoidosis Index (AMYLI) and amyloid light chain (AL) or inferior wall thickness (IWT) scores.

Patients in the original cohort were evaluated. 


\section{References}

1. Wechalekar AD, Gillmore JD, Hawkins PN. Systemic amyloidosis. Lancet. 2016;387:26412654.

2. Gillmore JD, Maurer MS, Falk RH, Merlini G, Damy T, Dispenzieri A, et al. Nonbiopsy diagnosis of cardiac transthyretin amyloidosis. Circulation. 2016;133:2404-12.

3. Falk RH, Alexander KM, Liao R, Dorbala S. AL (light-chain) cardiac amyloidosis: a review of diagnosis and therapy. J Am Coll Cardiol. 2016;68:1323-41.

4. Vergaro G, Aimo A, Barison A, Genovesi D, Buda G, Passino C, et al. Keys to early diagnosis of cardiac amyloidosis: red flags from clinical, laboratory and imaging findings. Eur J Prev Cardiol. 2019 Oct 4 [Epub ahead of print].

5. Boldrini M, Cappelli F, Chacko L, Baggiano A, Whelan C, Quarta C, et al. Multiparametric echocardiography scores for the diagnosis of cardiac amyloidosis. JACC Cardiovasc Imaging. 2020;13:909-20.

6. Park JH, Marwick TH. Use and limitations of E/e' to assess left ventricular filling pressure by echocardiography. J Cardiovasc Ultrasound. 2011;19:169-73.

7. Shephard DA. The 1975 Declaration of Helsinki and consent. Can Med Assoc J. 1976;115:1191-2.

8. Baggiano A, Boldrini M, Martinez-Naharro A, Kotecha T, Petrie A, Rezk T, et al. Noncontrast magnetic resonance for the diagnosis of cardiac amyloidosis. JACC Cardiovasc Imaging. 2020;13:69-80.

9. Deeks JJ, Altman DJ. Diagnostic tests 4: likelihood ratios. BMJ. 2004;329:168-9.

10. Tahir UA, Doros G, Kim JS, Connors LH, Seldin DC, Sam F. Predictors of mortality in light chain cardiac amyloidosis with heart failure. Sci Rep. 2019;9:8552.

11. Pozo E, Kanwar A, Deochand R, Castellano JM, Naib T, Pazos-López P, et al. Cardiac magnetic resonance evaluation of left ventricular remodelling distribution in cardiac amyloidosis. Heart. 2014;100:1688-1695.

12. Martinez-Naharro A, Treibel TA, Abdel-Gadir A, Bulluck H, Zumbo G, Knight DS, et al. Magnetic resonance in transthyretin cardiac amyloidosis. J Am Coll Cardiol. 2017;70:466477. 
13. Lavatelli F, Albertini R, Di Fonzo A, Palladini G, Merlini G. Biochemical markers in early diagnosis and management of systemic amyloidoses. Clin Chem Lab Med. 2014;52:151731.

This article is protected by copyright. All rights reserved 
Table 1. Derivation cohort.

\begin{tabular}{|c|c|c|c|c|}
\hline 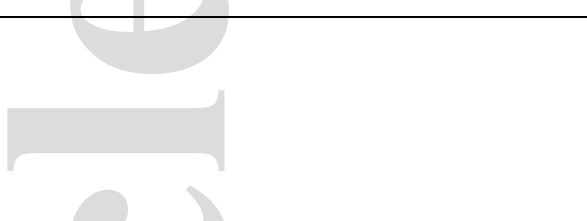 & $\begin{array}{l}\text { All patients } \\
\qquad \mathbf{n}=\mathbf{2 5 1}\end{array}$ & $\begin{array}{c}\text { Referred by } \\
\text { hematologists } \\
\text { n=67 }(27 \%)\end{array}$ & $\begin{array}{l}\text { Unexplained } \\
\text { hypertrophy } \\
\text { n=184 }(73 \%)\end{array}$ & $\mathbf{p}$ \\
\hline Men, n (\%) & $159(63)$ & $36(53)$ & $123(67)$ & 0.056 \\
\hline Age (years) & $79(74-84)$ & $74(65-78)$ & $81(77-85)$ & $<0.001$ \\
\hline $\mathrm{BMI}\left(\mathrm{kg} / \mathrm{m}^{2}\right)$ & $26.8(23.6-29.3)$ & $25.0(22.2-29.2)$ & $27.0(24.0-29.4)$ & 0.072 \\
\hline $\mathrm{SAP}(\mathrm{mmHg})$ & $130(120-150)$ & $125(110-140)$ & $135(120-150)$ & 0.005 \\
\hline $\mathrm{DAP}(\mathrm{mmHg})$ & $75(70-80)$ & $70(65-80)$ & $75(70-82)$ & 0.010 \\
\hline $\mathrm{eGFR}\left(\mathrm{mL} / \mathrm{min} / 1.73 \mathrm{~m}^{2}\right)$ & $58(41-79)$ & $62(39-81)$ & $57(41-76)$ & 0.306 \\
\hline NT-proBNP (ng/L) & $2662(985-7360)$ & $3525(627-10478)$ & $2512(1025-6466)$ & 0.294 \\
\hline \multicolumn{5}{|l|}{ 2D echo } \\
\hline LVEDVi $\left(\mathrm{mL} / \mathrm{m}^{2}\right)$ & $58(46-72)$ & $54(42-70)$ & $59(47-72)$ & 0.116 \\
\hline LVESVi $\left(\mathrm{mL} / \mathrm{m}^{2}\right)$ & $24(19-34)$ & $21(17-32)$ & $25(20-34)$ & 0.050 \\
\hline $\operatorname{LVMI}\left(\mathrm{g} / \mathrm{m}^{2}\right)$ & $147(120-180)$ & $129(105-155)$ & $153(125-183)$ & $<0.001$ \\
\hline RWT & $0.52(0.44-0.65)$ & $0.52(0.43-0.65)$ & $0.52(0.45-0.65)$ & 0.548 \\
\hline $\operatorname{LVEF}(\%)$ & $47(36-58)$ & $52(40-60)$ & $45(36-56)$ & 0.009 \\
\hline $\begin{array}{l}\text { LVEF }<40 \%, 40-49 \%, \geq 50 \%, \\
\text { n }(\%)\end{array}$ & $\begin{array}{l}83,54,106 \\
(33,22,42)\end{array}$ & $\begin{array}{c}14,16,35 \\
(21,24,52)\end{array}$ & $\begin{array}{c}69,38,71 \\
(38,21,39)\end{array}$ & $\mathbf{0 . 0 3 9}$ \\
\hline GLS (\%) & $-13(-17 /-8)$ & $-14(-17 /-11)$ & $-12(-16 /-8)$ & 0.105 \\
\hline $\mathrm{SAB}$ & $1.27(0.77-1.92)$ & $1.46(0.83-1.93)$ & $1.26(0.71-1.94)$ & 0.300 \\
\hline E/e' ratio & $12.6(9.3-17.0)$ & $13.1(8.9-21.8)$ & $12.6(9.3-16.6)$ & 0.466 \\
\hline TAPSE $(\mathrm{mm})$ & $18(15-22)$ & $18(16-22)$ & $17(15-21)$ & 0.295 \\
\hline AMYLI & $6.8(4.3-10.8)$ & $6.8(4.0-12.4)$ & $6.8(4.5-10.7)$ & 0.823 \\
\hline
\end{tabular}

Estimated glomerular filtration rate (eGFR) was calculated through the Chronic Kidney Disease Epidemiology Collaboration equation. AMYLI, AMYLoidosis Index; BMI, body mass index; DAP, diastolic arterial pressure; GLS, global longitudinal strain; LVEDVi, left ventricular end-diastolic volume index; LVEF, left ventricular ejection fraction; LVESVi, left ventricular end-systolic volume index; LVMI, left ventricular mass index; NT-proBNP, Nterminal fraction of pro-B-type natriuretic peptide; RWT, relative wall thickness; SAB, 
systolic apex-to-base ratio; SAP, systolic arterial pressure; TAPSE, tricuspid annular plane systolic excursion. 
Table 2. Validation cohort.

\begin{tabular}{|c|c|c|c|c|}
\hline 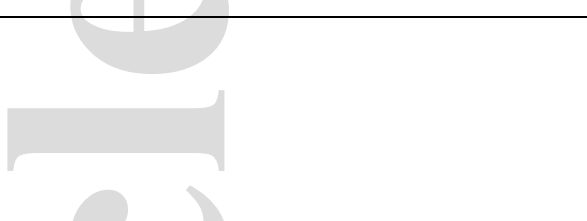 & $\begin{array}{l}\text { All patients } \\
n=691\end{array}$ & $\begin{array}{c}\text { Referred by } \\
\text { hematologists } \\
\text { n=408 }(59 \%)\end{array}$ & $\begin{array}{l}\text { Unexplained } \\
\text { hypertrophy } \\
\text { n=283 }(41 \%)\end{array}$ & $\mathbf{p}$ \\
\hline Men, n (\%) & $462(67)$ & $233(57)$ & $229(81)$ & $<0.001$ \\
\hline Age (years) & $69(60-76)$ & $66(58-72)$ & $75(65-80)$ & $<0.001$ \\
\hline $\mathrm{BMI}\left(\mathrm{kg} / \mathrm{m}^{2}\right)$ & $25.8(23.4-29.5)$ & $25.3(22.9-29.0)$ & $26.8(24.3-30.0)$ & $<0.001$ \\
\hline $\mathrm{SAP}(\mathrm{mmHg})$ & $123(111-140)$ & $122(110-135)$ & $125(114-143)$ & 0.025 \\
\hline $\mathrm{DAP}(\mathrm{mmHg})$ & $75(68-81)$ & $74(68-81)$ & $75(70-82)$ & 0.047 \\
\hline $\mathrm{eGFR}\left(\mathrm{mL} / \mathrm{min} / 1.73 \mathrm{~m}^{2}\right)$ & $71(47-91)$ & $75(50-94)$ & $63(41-84)$ & $<0.001$ \\
\hline NT-proBNP (ng/L) & $1777(507-4242)$ & $1655(426-3878)$ & $1844(746-4967)$ & 0.116 \\
\hline \multicolumn{5}{|l|}{ 2D echo } \\
\hline LVEDVi $\left(\mathrm{mL} / \mathrm{m}^{2}\right)$ & $38(29-49)$ & $40(29-50)$ & $37(29-47)$ & 0.415 \\
\hline LVESVi $\left(\mathrm{mL} / \mathrm{m}^{2}\right)$ & $17(12-25)$ & $17(12-25)$ & $17(13-25)$ & 0.372 \\
\hline $\operatorname{LVMI}\left(\mathrm{g} / \mathrm{m}^{2}\right)$ & $148(114-188)$ & $136(105-180)$ & $166(129-207)$ & $<0.001$ \\
\hline RWT & $0.62(0.49-0.78)$ & $0.59(0.47-0.73)$ & $0.68(0.53-0.82)$ & $<0.001$ \\
\hline $\operatorname{LVEF}(\%)$ & $56(47-63)$ & $57(50-64)$ & $54(43-61)$ & $<0.001$ \\
\hline $\begin{array}{l}\text { LVEF }<40 \%, 40-49 \%, \geq 50 \%, \\
\text { n }(\%)\end{array}$ & $\begin{array}{l}92,109,474 \\
(13,16,69)\end{array}$ & $\begin{array}{l}38,59,299 \\
(9,15,73)\end{array}$ & $\begin{array}{l}54,50,175 \\
(19,18,62)\end{array}$ & $<0.001$ \\
\hline GLS (\%) & $-12(-17 /-9)$ & $-14(-18 /-10)$ & $-11(-15 /-8)$ & $<0.001$ \\
\hline $\mathrm{SAB}$ & $3.09(2.00-6.00)$ & $2.86(2.00-5.33)$ & $3.30(1.97-6.54)$ & 0.340 \\
\hline E/e' ratio & $12.4(8.3-17.6)$ & $10.7(8.0-16.0)$ & $13.8(9.1-20.1)$ & $<0.001$ \\
\hline TAPSE (mm) & $18(14-22)$ & $19(15-22)$ & $18(14-22)$ & 0.124 \\
\hline AMYLI & $7.6(4.3-12.0)$ & $6.5(3.9-11.3)$ & $10.0(5.5-14.9)$ & $<0.001$ \\
\hline
\end{tabular}

Estimated glomerular filtration rate (eGFR) was calculated through the Chronic Kidney Disease Epidemiology Collaboration equation. AMYLI, AMYLoidosis Index; BMI, body mass index; DAP, diastolic arterial pressure; GLS, global longitudinal strain; LVEDVi, left ventricular end-diastolic volume index; LVEF, left ventricular ejection fraction; LVESVi, left ventricular end-systolic volume index; LVMI, left ventricular mass index; NT-proBNP, Nterminal fraction of pro-B-type natriuretic peptide; RWT, relative wall thickness; SAB, 
systolic apex-to-base ratio; SAP, systolic arterial pressure; TAPSE, tricuspid annular plane systolic excursion. 
Table 3. AMYLI score values to rule-out and rule-in cardiac amyloidosis.

\begin{tabular}{|c|c|c|c|c|c|c|c|c|c|c|c|c|c|}
\hline Patient subset & Score values & \multicolumn{6}{|c|}{ Original cohort } & \multicolumn{6}{|c|}{ Validation cohort } \\
\hline 3 & & $\begin{array}{c}\text { Sensitivity } \\
(\%)\end{array}$ & $\begin{array}{c}\text { Specificity } \\
(\%)\end{array}$ & $\begin{array}{l}\text { PPV } \\
(\%)\end{array}$ & $\begin{array}{l}\text { NPV } \\
(\%)\end{array}$ & LR+ & LR- & $\begin{array}{c}\text { Sensitivity } \\
(\%)\end{array}$ & $\begin{array}{c}\text { Specificity } \\
(\%)\end{array}$ & $\begin{array}{l}\text { PPV } \\
(\%)\end{array}$ & $\begin{array}{l}\text { NPV } \\
(\%)\end{array}$ & $\begin{array}{l}\text { LR+ } \\
(\%)\end{array}$ & $\begin{array}{l}\text { LR- } \\
(\%)\end{array}$ \\
\hline \multirow[t]{2}{*}{ Whole cohort } & Rule-out: 2.22 & 100 & 5 & 45 & 100 & 1.1 & 0.0 & 97 & 0 & 83 & 0 & 0.0 & $\infty$ \\
\hline & Rule-in: 26.79 & 4 & 99 & 80 & 57 & 5.0 & 1.0 & 2 & 99 & 93 & 17 & 2.0 & 1.0 \\
\hline \multirow{2}{*}{$\begin{array}{l}\text { Referred by } \\
\text { hematologists }\end{array}$} & Rule-out: 2.36 & 100 & 6 & 58 & 100 & 1.1 & 0.0 & 95 & 0 & 94 & 0 & 0.95 & $\infty$ \\
\hline & Rule-in: 12.97 & 39 & 94 & 90 & 55 & 7.0 & 0.6 & 20 & 100 & 100 & 7 & $\infty$ & 0.8 \\
\hline \multirow{2}{*}{$\begin{array}{l}\text { Unexplained } \\
\text { hypertrophy }\end{array}$} & Rule-out: 2.22 & 100 & 3 & 47 & 100 & 1.0 & 0.0 & 97 & 0 & 68 & 0 & 1.0 & $\infty$ \\
\hline & Rule-in: 9.63 & 51 & 83 & 71 & 67 & 3.0 & 0.6 & 59 & 66 & 78 & 42 & 1.7 & 0.6 \\
\hline
\end{tabular}

Optimal rule-out cut-offs have a likelihood ratio - (LR-: [1- sensitivity]/specificity) <0.1, while optimal rule-in cut-offs have a LR+ (sensitivity/[1-specificity]) $>10$. LR were equal to infinite when the denominator was $=0$. The rule-in cut-offs selected were those with the highest LR+ value in the original cohort. NPV, negative predictive value; PPV, positive predictive value. 


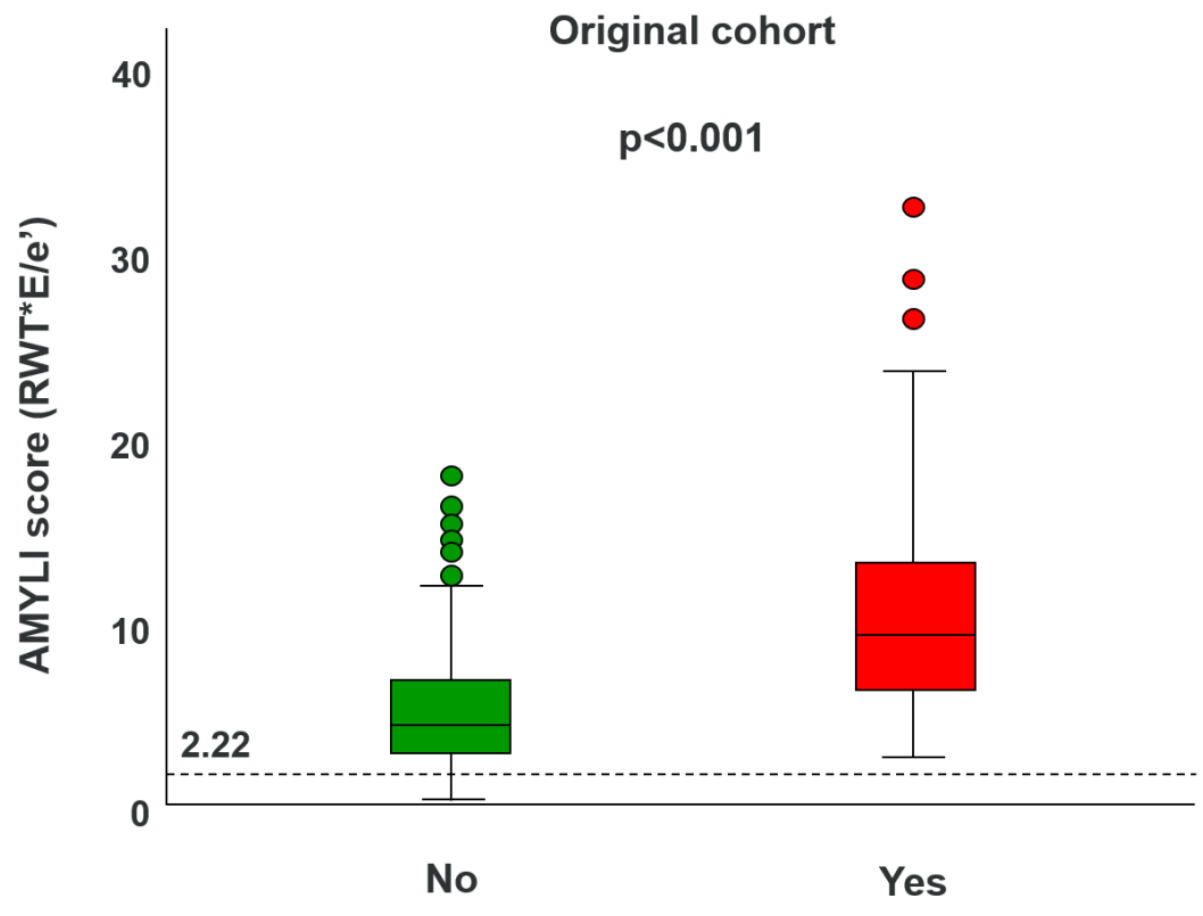

Cardiac amyloidosis

eci_13449_f1.tif 


\section{Hematology subset}

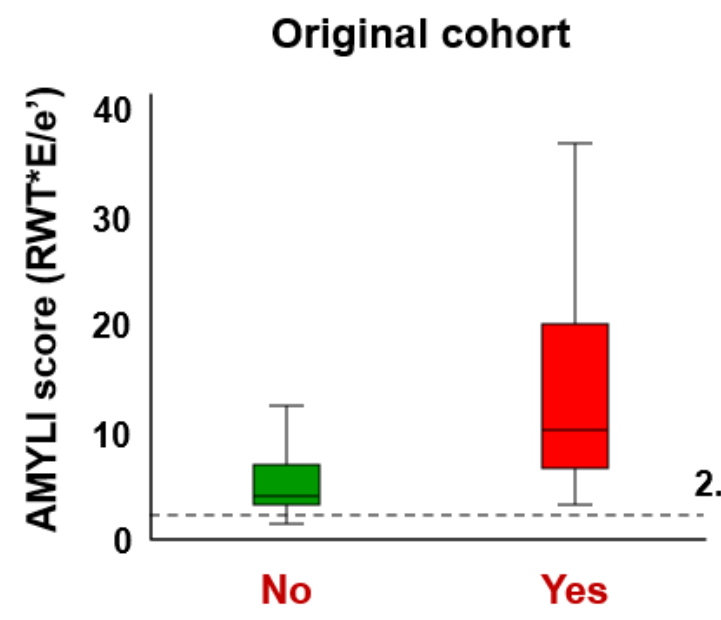

AL

Validation cohort

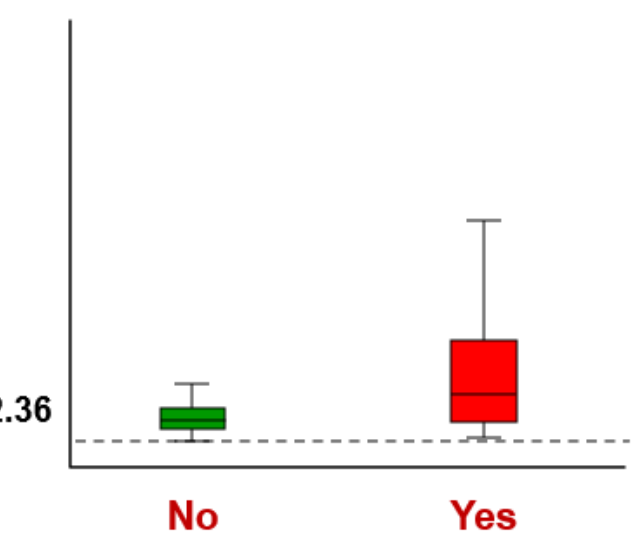

AL

Hypertrophy subset

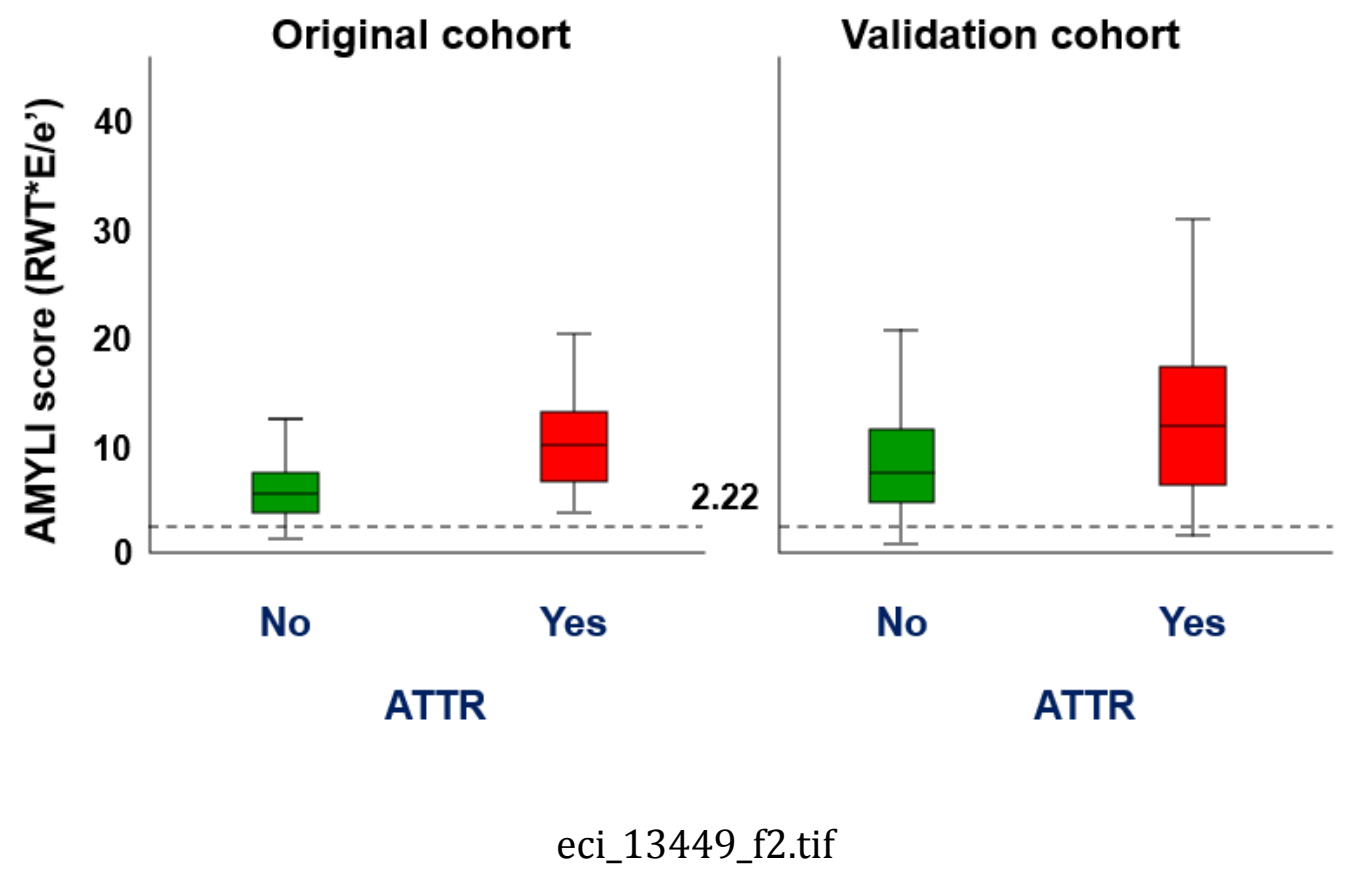

This article is protected by copyright. All rights reserved 
Original cohort

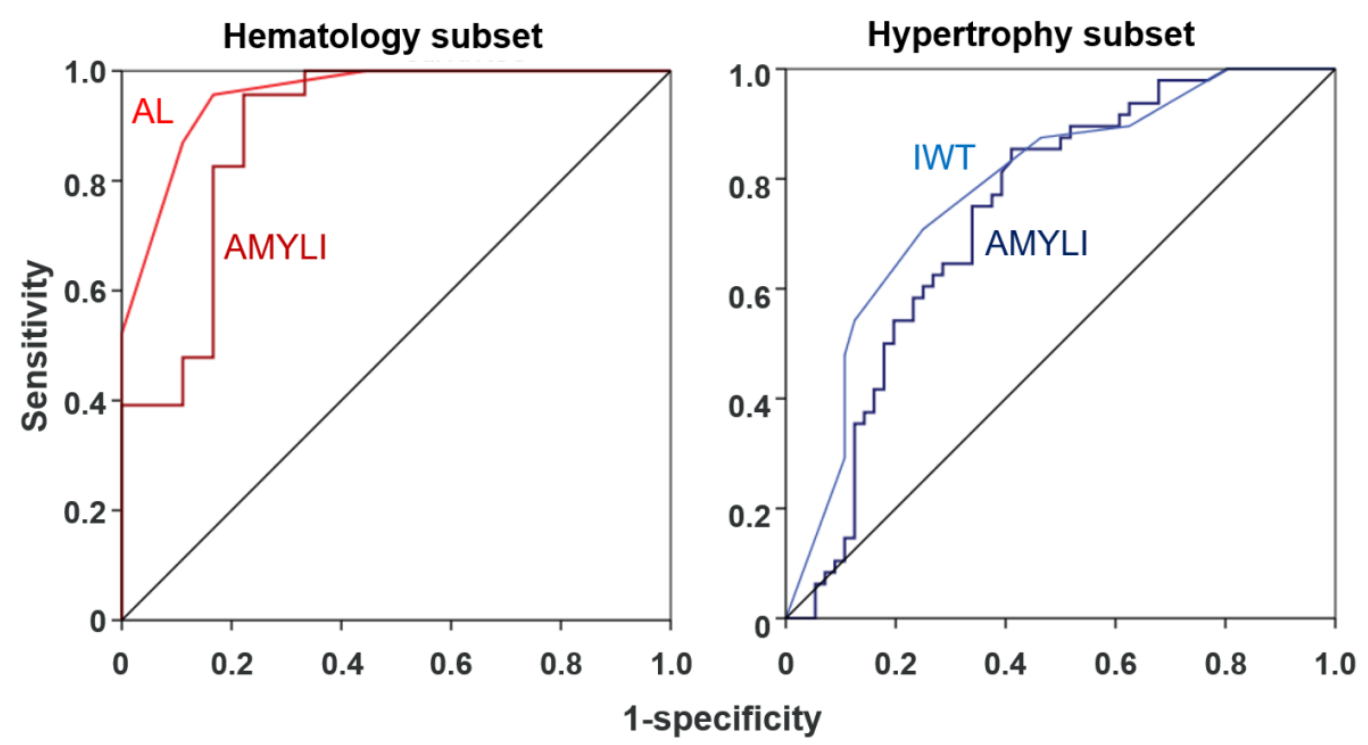

AL score ( $n=41,61 \%)$ : AUC 0.955

IWT score $(n=104,57 \%)$ : AUC 0.803

AMYLI ( $n=67,100 \%)$ : AUC 0.889

AMYLI $(n=184,100 \%)$ : AUC 0.746 $\mathrm{p}=0.095$

$\mathrm{p}=0.347$

eci_13449_f3.tif

This article is protected by copyright. All rights reserved 